\title{
VENTAJAS DE LA GAMIFICACIÓN EN EL ÁMBITO DE LA EDUCACIÓN FORMAL EN ESPAÑA. UNA REVISIÓN BIBLIOGRÁFICA EN EL PERÍODO DE 2015-2020
}

\author{
Eva Pérez Gallardo \\ Felipe Gértrudix-Barrio \\ Universidad de Castilla La Mancha
}

\begin{abstract}
RESUMEN: Algunos principios de intervención educativa, como es el aprendizaje lúdico, han ido virando hacia propuestas más enriquecedoras como la gamificación. Este término, con auge en el campo educativo, profesional y empresarial, implica el uso de mecánicas propias de juegos en contextos habitualmente no lúdicos. En este contexto, el objetivo del trabajo es analizar los efectos positivos que genera la aplicación de técnicas de gamificación en las aulas educativas. El método ha sido una revisión bibliográfica de literatura de 39 artículos publicados en acceso abierto, de enero de 2015 a abril de 2020, en las bases de datos Web of Science (SSCI) y Scopus, mediante el sistema de validación de pertinencia, inclusión y exclusión, calidad/validez de los estudios y descripción de datos, según los criterios de la Universidad de York (PRISMA). En el análisis se han buscado los códigos del discurso y las relaciones entre las variables estudiadas con el fin de conocer la relación de las dinámicas que interactúan entre las mismas. Como inferencia de los resultados se evidencia el impacto positivo de la gamificación en el proceso de enseñanza-aprendizaje, siendo la motivación y el rendimiento académico los aspectos más destacables.
\end{abstract}

PALABRAS CLAVE: educación formal, gamificación, motivación, rendimiento académico.

\section{ADVANTAGES OF GAMIFICATION IN THE FIELD OF FORMAL EDUCATION IN SPAIN. A BIBLIOGRAPHIC REVIEW IN THE PERIOD} 2015-2020

\footnotetext{
ABSTRACT: Some principles of educational intervention, such as playful learning, have been turning towards more enriching proposals such as gamification. This term, booming in the educational, professional and business
} 
fields, implies the use of game mechanics in habitually non-recreational contexts. In this context, the objective of the work is to analyze the positive effects generated by the application of gamification techniques in educational classrooms. The method was a bibliographic review of 39 articles published in open access, from January 2015 to April 2020, in the Web of Science (SSCI) and Scopus databases using the system of validation of relevance, inclusion and exclusion, quality / validity of studies and description of data, according to the criteria of the University of York (PRISMA). In the analysis, the codes of the discourse and the relationships between the variables studied have been searched in order to know the relationship of the dynamics that interact between them. As an inference from the results, the positive impact of gamification in the teaching-learning process is evidenced, with motivation and academic performance being the most notable aspects.

KEYWORDS: Formal education, gamification, motivation, academic performance.

Recibido:16/06/2020

Aceptado: 20/02/2021

Correspondencia: Felipe Gértrudix-Barrio, Facultad de Educación, Universidad de Castilla La Mancha, Avenida Carlos III, s/n, 45071 Toledo. Email: felipe.gertrudix@ uclm.es

\section{INTRODUCCIÓN}

Los constantes cambios del entorno social repercuten directamente en el sistema educativo, empujando a la escuela a renovarse cada día para adaptarse a las nuevas demandas y ofrecer su mejor versión. Esto induce a una transformación del enfoque pedagógico y de las metodologías, dando paso cada vez a un mayor protagonismo del alumnado.

En palabras de Ibáñez et al. (2008), los estudiantes perciben la educación como una mercancía que se adquiere y consume. Esperan que su aproximación al conocimiento sea rápido, sencillo y entretenido; minimizando el tiempo de estudio e incrementado los resultados. Se declinan por la práctica frente a la teoría, las tareas grupales en lugar de las individuales y la información en formato digital frente la impresa. De este modo, los centros educativos, en su esfuerzo por mejorar los procesos de enseñanza y aprendizaje, buscan alternativas e introducen continuamente propuestas innovadoras en las aulas. En este contexto se encuadra la técnica de la gamificación, que tiene su origen en el juego.

Como sabemos, el juego es un elemento indisociable de la vida del niño, el cual constituye un factor fundamental para el aprendizaje en la primera infancia, siendo una de las vías privilegiadas de acceso al conocimiento que permite descubrir, explorar, comprender y consolidar (Paniagua y Palacios, 2008); una actividad natural del hombre, y especialmente importante en la vida de los niños, que estimula el acerca- 
miento al mundo que le rodea y la comprensión de la realidad (Llull y García, 2009). Favorece, además, el desarrollo de la imaginación, la atención, la comunicación y la libertad (Gómez, 2012), la interacción social con los iguales, así como el desarrollo físico, intelectual, emocional, social y moral a todas las edades, adaptándose a la realidad externa (Gallardo y Gallardo, 2018).

Si bien es cierto que la gamificación tiene un componente lúdico, se trata de algo más que un juego. Concretamente, el término gamificación es un anglicismo que deriva de la raíz "game", que significa juego, y el sufijo "ificaction", que alude a un proceso productivo, es decir, se podría entender como una "puesta en juego" (Pascuas et al., 2017). Según Carreras (2017), de manera análoga, su acepción en castellano es ludificación, del latín "ludus", "ludure" (juego, jugar). El término aparece por primera vez en 2002 acuñado por el programador británico de software Nick Pelling (Oliva, 2016; Carreras, 2017; Vergara et al., 2019), y rápidamente fue ganando peso en el mundo del videojuego y del marketing, hasta que en 2008 se le asigna el sentido con el que hoy lo conocemos, Ilegado a campos muy diversos, como el medio ambiente, la formación en empresas, la salud y la educación.

Centrándonos en el terreno educativo, la gamificación se concibe como el diseño de escenarios de aprendizaje, constituidos por actividades dinámicas e ingeniosas que incitan a la resolución de tareas de forma innovadora y colaborativa, impulsando a los estudiantes a la superación de retos y a la consecución de más y mejores niveles de competencia (Lee y Hammer, citado en Villalustre y del Moral, 2015). La eficaz vinculación entre los elementos del juego y la acción educativa despertará el interés del alumnado por el aprendizaje y conducirá al desarrollo de su potencial (Oliva, 2016).

En cualquier caso, podemos decir que la gamificación se traduce en una actividad que adopta las estrategias y mecánicas propias del juego, pero se desarrolla en un contexto formal no lúdico. Estas prácticas buscan la implicación de los participantes y están orientadas hacia la consecución de un fin específico (modificar conductas, adquirir conocimientos, mejorar habilidades), por tanto, promueven una transformación en el sujeto al mismo tiempo que generan una experiencia gratificante. Para llegar a la meta han de afrontar desafíos y respetar las reglas que se dan en cualquier juego convencional, actuando, en ocasiones, de manera cooperativa. La retroalimentación y los premios o compensaciones adquieren un papel decisivo en este proceso.

La influencia que tiene sobre el individuo es innegable pues, como puntualizan Villalustre y del Moral (2015), los usuarios se convierten en jugadores activos que se sumergen en entornos lúdicos en los que se enfrentan a misiones atractivas que les envuelven emocionalmente, logrando incrementar su implicación. En algunos casos, las dinámicas son similares a las de los juegos competitivos por lo que su diseño y aplicación en entornos educativos, debe tratarse desde una perspectiva pedagógica y didáctica (Marczewski, 2015).

Werbach y Hunter (2012), identifican tres categorías de elementos básicos en los sistemas gamificados:

1. Las dinámicas constituyen la estructura implícita del juego.

2. Las mecánicas son los procesos que estimulan el desarrollo del juego.

3. Los componentes pueden definirse como implementaciones concretas de las dinámicas y las mecánicas, es decir, la materialización específica de estas últimas. 
Gráfico 1. Claves para entender la gamificación

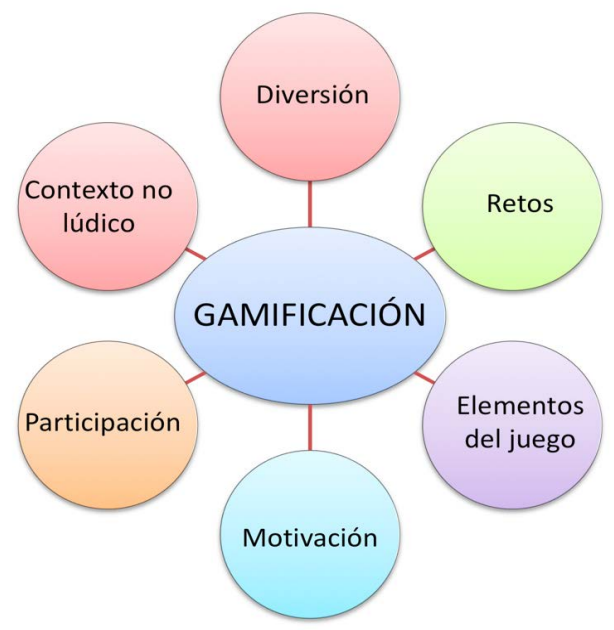

Además, organizan estos tres elementos jerárquicamente, haciendo explícita la interrelación entre ellos. En la cúspide las dinámicas, los aspectos generales que no se introducen en el juego y cuyo diseño y gestión resulta trascendental. En la parte central las mecánicas, reconocidas como los procesos que dirigen la acción y generan fidelización. En la base los componentes, que hacen posible el desarrollo de las dinámicas y mecánicas. No obstante, difícilmente encontraremos un proyecto gamificado que incluya todos los elementos a la vez.

Gráfico 2. Pirámide de Werbach y Hunter. Fuente: elaborado a partir de Werbach y Hunter (2012)

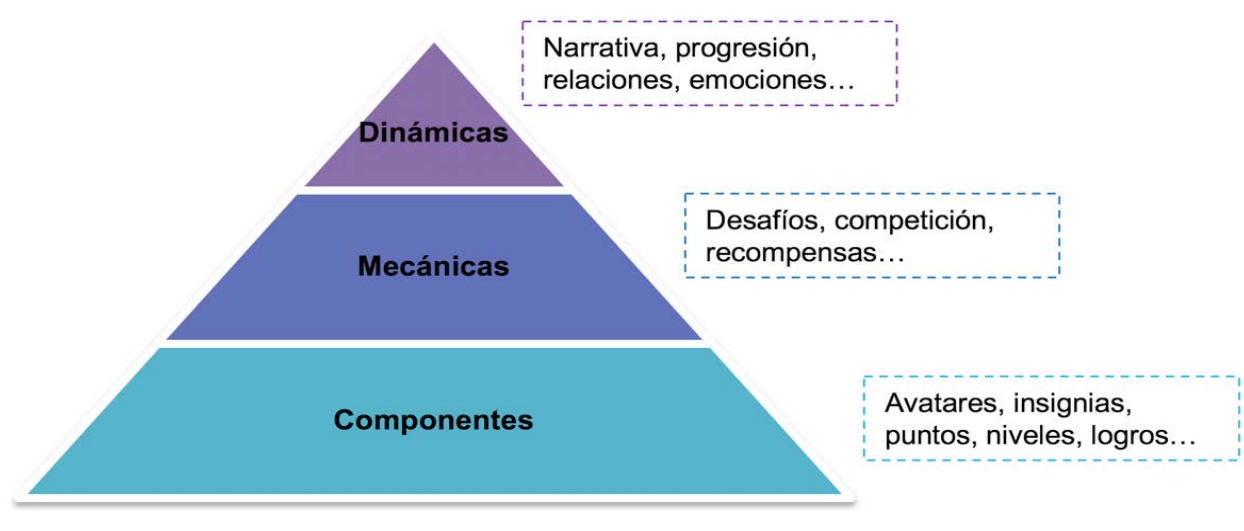

Dada su expansión y alcance en los últimos años, el presente trabajo se centra en el análisis sobre la viabilidad de la gamificación como recurso en el marco de la educación formal, entendiendo a esta como el tipo de educación regulada y planificada 
que nos aproxima a un paradigma de estudio concretizado en un currículo oficial para ser titulado (Colom, 2005), haciendo hincapié en sus principales beneficios como estrategia con el fin de ratificar su valor educativo.

\section{ANTECeDentes}

En el ámbito de la educación existen algunas revisiones sobre la aplicación de la gamificación en el aula y su impacto en la formación. Un ejemplo específico es la investigación de Ortiz-Colón et al. (2018), que arroja información sobre los beneficios y dificultades que generan en los estudiantes los procesos de gamificación. Entre sus hallazgos destacan los niveles de motivación y su incidencia en el compromiso; la inmersión, que facilita la anticipación y planificación de situaciones; y la socialización.

Desde una perspectiva más amplia, Pascuas et al. (2017) analizan el impacto de la gamificación en la formación en el sector educativo y empresarial. Aunque se debe ajustar una buena planificación en la implementación de actividades gamificadas y que estas sean justificadas (Burke, 2014), los datos obtenidos en educación denotan mejoras en parámetros como la motivación, la participación, la concentración y los cambios de comportamiento. Además, destacan la posibilidad de abandonar la rutina en beneficio de un ambiente más cómodo y actualizado, implementando las TIC como medio para poner a disposición del alumnado los contenidos de aprendizaje.

Centrado en el área de Educación Física, Escaravajal y Martín-Acosta (2019) apuntan hacia la consolidación de los objetivos y el aprendizaje significativo como las variables más repetidas en los trabajos de gamificación para esta disciplina. Seguidamente destacan la potenciación de la motivación y la satisfacción de los estudiantes. Con todo, proponen la integración de esta metodología en las aulas para avanzar y mejorar la enseñanza.

En el marco de la educación superior, Lozada-Ávila y Betancur-Gómez (2017), determinan una falta de unificación en los resultados, particularmente en las calificaciones, pues la gamificación no siempre incurre en una mejora del desempeño ni una mayor satisfacción. Por otro lado, subrayan que la tecnología es una alidada de este método, debido a la mayor interacción social; aun así, no tiene por qué formar parte de todas las propuestas.

En la misma etapa educativa, Prieto (2020) examina la motivación y el rendimiento de los universitarios derivado de la implementación de propuestas de gamificación. Los resultados son favorables para ambos factores, pero en algunos los estudios no perciben mejoras en la motivación intrínseca, probablemente por un diseño inadecuado o la escasa duración de la propuesta (Marczewski, 2015). Así, para alcanzar el efecto esperado, establecen unas pautas como: combinar distintas mecánicas, especificar el objetivo, explicar previamente el sistema de puntos, medir la carga de trabajo y valorar las condiciones personales de los destinatarios (Zichermann y Cunningham, 2011; Zichermann y Linder, 2013).

Igualmente, Alomari et al. (2019) concluyen que las técnicas gamificadas son un recurso muy potente para promover la motivación, el compromiso y el rendimiento. 
Su logro reside, en cierta medida, en la información que se ofrece a los estudiantes sobre la propuesta y en la valoración de los elementos que influyen en el proceso de aprendizaje y no en el contexto en sí mismo.

Atendiendo a la educación gerencial, Rodrigues et al. (2019) identifica cuatro variables fundamentales en las experiencias gamificadas, que ordenadas de manera descendente son: motivación, aprendizaje, actitudes y flujo. Entre ellas se establece una relación causa-efecto, especialmente en el caso de la motivación y el aprendizaje, que es la construcción que aparece con más frecuencia en las investigaciones.

Tomando como referencia estos estudios, y teniendo en cuenta que en algunos documentos de ellos se refieren a la falta de evidencia empírica, orientamos nuestra investigación hacia el análisis de las principales ventajas de la gamificación en el panorama educativo español con el fin de dar respuesta a las siguientes cuestiones:

- ¿Cuál es el alcance real de la gamificación en la educación formal?

- ¿Puede la gamificación, como estrategia educativa, introducir mejoras en el proceso de enseñanza y aprendizaje y en los resultados?

- ¿Qué factores o variables son potenciados en los sistemas gamificados?

\section{Objetivos}

Como objetivo principal se plantea explorar las repercusiones educativas de las experiencias gamificadas como estrategia para mejorar la efectividad de los procesos de enseñanza y aprendizaje en la educación formal. Como variables de investigación se han establecido las siguientes: 1) Motivación, 2) Compromiso, 3) Cohesión grupal, 4) Emoción y 5) Rendimiento académico.

A partir de este objetivo prioritario se extraen los siguientes objetivos específicos:

1. Identificar las principales ventajas de la gamificación como técnica educativa y su influencia en el aprendizaje y desarrollo del alumnado.

2. Establecer la relación de las dinámicas que interactuarían entre las cinco variables estudiadas: motivación; compromiso, cohesión grupal, emoción y rendimiento académico.

3. Establecer cuál es el potencial innovador de la gamificación en el proceso de enseñanza y aprendizaje como factor para la transformación educativa.

\section{Método}

El enfoque metodológico ha sido el análisis cualitativo de revisión bibliográfica, según Piantanida y Garman (1999) y Savin-Baden y Major (2013) con el fin de identificar y analizar los efectos positivos que genera la aplicación de técnicas de gamificación en las aulas educativas. Para ello se ha utilizado el software Atlas.ti.9. con la finalidad de encontrar aquellos factores comunes en las investigaciones más relevantes de modo que se establezca un posible paradigma respecto a las investigaciones sobre gamificación como transmisión de aprendizajes realizadas entre 2010-2020 en el contexto de España. 


\section{Estrategias de selección}

De acuerdo con estos planteamientos, los documentos que se han seleccionado y analizado pertenecen a revistas científicas recogidas en las bases de datos SCOPUS y en la Social Sciences Citation Index (SSCI) de Web Of Science (WOS), dado que son las principales fuentes de artículos de divulgación científica garantizando, con ello, una mayor influencia y accesibilidad a revistas y artículos (Granda-Orive et al., 2013).

Los criterios de selección que manejamos en la búsqueda son la validación de pertinencia, inclusión y exclusión, calidad/validez de los estudios y descripción de datos, han sido los declarados en PRISMA de la Universidad de York (2015):

- Datados en los últimos 5 años (hasta mayo de 2020), coincidiendo con el inicio de la expansión de la gamificación.

- Relevancia de los contenidos implícitos.

- Publicados en lengua inglesa o castellano.

- Acceso libre al texto completo en la base de datos.

Los términos de recuperación empleados inicialmente fueron: "gamificación" y "educación primaria", sin embargo, dada la escasez de paper dedicados a esta etapa, ampliamos nuestro horizonte abordando la gamificación en cualquier etapa educativa.

\section{Selección de artículos}

La selección ha seguido los pasos siguientes (ver Gráfico 3):

1) Haciendo uso de los términos escogidos, el 5 de mayo de 2020 se llevó acabo la búsqueda de artículos con los siguientes criterios de exclusión:

- Los documentos con una antigüedad posterior al año 2010, pues ambas temáticas -educación y gamificación- están en constante actualización y un artículo anterior podría contener información desfasada y obsoleta.

- Las referencias que no estaban escritos en inglés y español fueron descartadas.

En esta primera búsqueda se obtuvieron un total de 368 documentos en Scopus y 533 en Wos.

2) La segunda búsqueda se hizo en función de:

- El tipo de documento; aquellos que no eran artículos fueron excluidos.

- Su accesibilidad; aquellos documentos que no estaban en Revistas Open Acces se eliminaron.

- El contexto de la investigación; se eliminaron aquellos que no estaban vinculados directamente con España.

- Una vez seleccionados dichos criterios, el resultado de la búsqueda se redujo a 160 documentos. Estos estudios fueron procesados a través de Mendeley para eliminar duplicados, dando un resultado final para su posterior selección de 111 artículos. Finalmente, el tamaño muestral quedó reducido a 39 artículos, los cuales están identificados con un asterisco en referencias bibliográficas. 
Gráfico 3. PRISMA. Diagrama de flujo de la selección de documentos

Búsqueda en bases de datos: WOS y Scopus

Términos de recuperación: "gamification" and "education"

Textos en inglés y en castellano con acceso
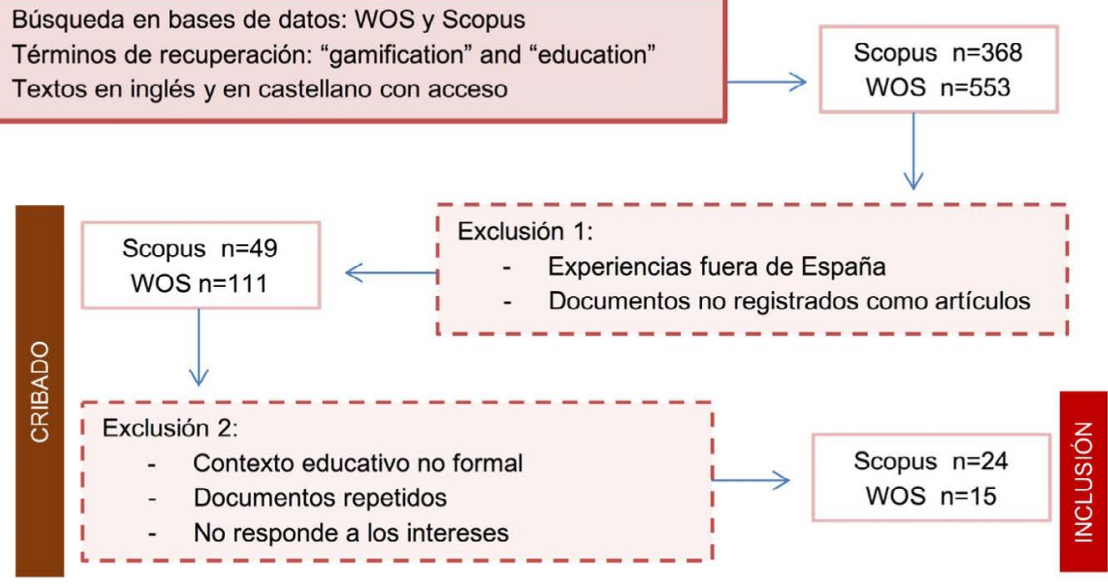

Gráfico 4. $N^{o}$ de artículos seleccionados por año

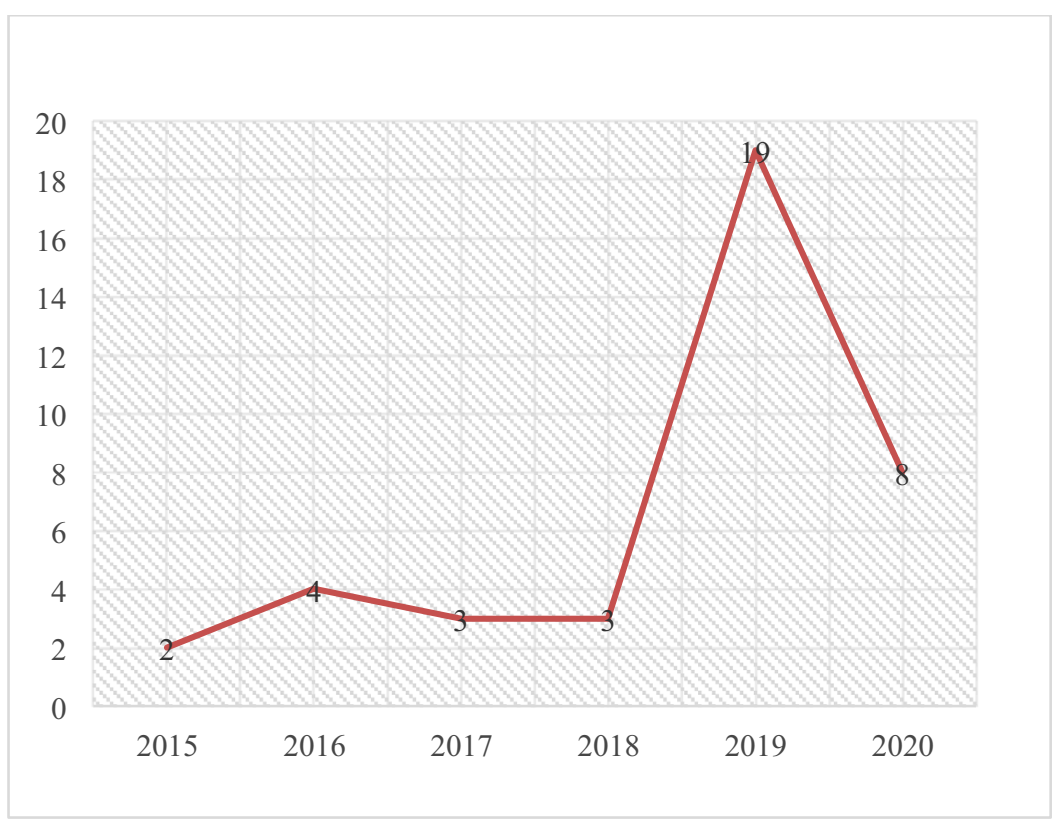

Es importante destacar cómo el interés en torno a la gamificación en educación se ha producido especialmente en los últimos años (ver Gráfico 4). 


\section{Análisis de los resultados}

En un primer momento se realizó un análisis detallado (microanálisis) con el fin de definir las unidades de texto y extraer los códigos sustanciales. En un nivel de abstracción superior se marcaron los códigos teóricos, y se clasificaron según sus peculiaridades, comparando las similitudes y diferencias de estos conceptos para la elaboración de las categorías.

Para el hallazgo de la categoría central se descartaron las propiedades menos importantes para llegar a la saturación y poder conectar la teoría con los datos.

El proceso de la estrategia analítica cualitativa acometido fue el siguiente: a cada unidad de texto se le agregó una o varias etiquetas/marcas/claves que relacionaba el contenido de ese fragmento de texto con una categoría determinada. Se decidió utilizar códigos con abreviaturas de las palabras que correspondían a las categorías en las que se englobarían: motivación (M), compromiso/participación (CP), cohesión grupal/cooperación (CC), emociones $(\mathrm{E})$ y rendimiento académico (RA).

\section{Motivación (M)}

La motivación es un factor clave en los discursos acerca de la gamificación, ya sea en el campo educativo, empresarial, publicitario, etc.: un elemento para satisfacer las necesidades de motivación hacia una actividad" (Pascuas et al., 2017, p. 68).

Teniendo en cuenta las tasas de abandono y fracaso escolar que prevalecen en la actualidad, la motivación puede considerarse como uno de los principales retos a los que se enfrentan los docentes en el proceso de enseñanza y aprendizaje. Así, la gamificación como base de los programas educativos se convierte en un potente recurso para reducir el aburrimiento y el desinterés de los estudiantes (Ortiz-Colon et al., 2018).

Tradicionalmente, la motivación se ha seccionado en dos parcelas diferenciadas: la extrínseca y la intrínseca. La primera es externa al individuo, esto es, un incentivo exterior que empuja al sujeto a actuar de un modo determinado para alcanzar un premio o reconocimiento. La segunda se asocia a la satisfacción personal, orientada hacia la adquisición de conocimientos, mejora de habilidades o desarrollo de destrezas. Ambas deben estar presentes en los sistemas gamificados con un equilibrio armónico (Llorens et al., 2016; Castañeda-Vázquez et al. 2019).

Bajo el paraguas de las experiencias gamificadas encontramos que la motivación intrínseca se relaciona con el interés por aprender y la propia formación; mientras que la extrínseca está ligada a las recompensas, como los puntos, insignias o tablas de clasificación. De esta forma, el estudiante da muestras de esa motivación cuando decide comprometerse con una actividad, esforzándose y siendo persistente en su ejecución (García y Sevilla, 2019).

El éxito de la gamificación recae en parte en la motivación, por su carácter novedoso y lúdico. La posibilidad que se le brinda al alumnado para experimentar y descubrir cosas nuevas, al tiempo que se divierten y adquieren conocimientos, hacen de ella un aliado fundamental para la enseñanza y el aprendizaje (Manzano-León et al., 2020), convirtiendo ciertos temas calificados como tediosos en contenidos más asequibles y amenos (Álvaro-Tordesillas, et al., 2020). Es más, "cuando los estudiantes perciben el aprendizaje de un modo dinámico y envuelto en una narrativa atractiva, 
la motivación pasa a ser el motor del proceso educativo" (Manzano-León et al., 2020, p. 5). Así, se puede afirmar que el simple placer de jugar es un desencadenante más de la motivación intrínseca, frente a otras prácticas tradicionales en las que prevalece la motivación extrínseca (Ortega y Gómez, 2019).

En este sentido, los datos son concluyentes, la motivación es uno de los ejes que sustentan los sistemas gamificados, sin embargo, no todos los estudios coinciden en la presencia equitativa de sus dos dimensiones. Manzano-León et al. (2020) apuntan que la asunción de un rol o personaje incrementa la motivación interna del alumnado, y con ello su nivel de desempeño en las actividades.

Ibáñez et al. (2015) también analizan la determinación de la motivación, conviniendo que la estimulación de algunos estudiantes para participar en este tipo de propuestas se asocia a la recolección de insignias o el posicionamiento en las tablas de clasificación; al contrario de otros, que atribuyen su involucración al logro de hitos académicos y objetivos personales (Gómez-Carrasco et al., 2019; Pérez-López et al., 2019; Zamora-Polo et al., 2019; Díaz-Lauzurica y Moreno-Salinas; 2019).

En muchas de las experiencias desarrolladas en los distintos niveles educativos las TIC adquieren un papel decisivo, ya sea mediante videojuegos, plataformas educativas o redes sociales (de-Marcos et al., 2016; Capel et al., 2017; Gómez, 2019; Faya y Martín-Macho, 2019; Parra-González et al., 2020). La condición de "nativo digital" de los estudiantes convierten a estos recursos en un elemento motivador y un medio para fomentar su implicación.

Gráfico 5. Factores asociados a la motivación

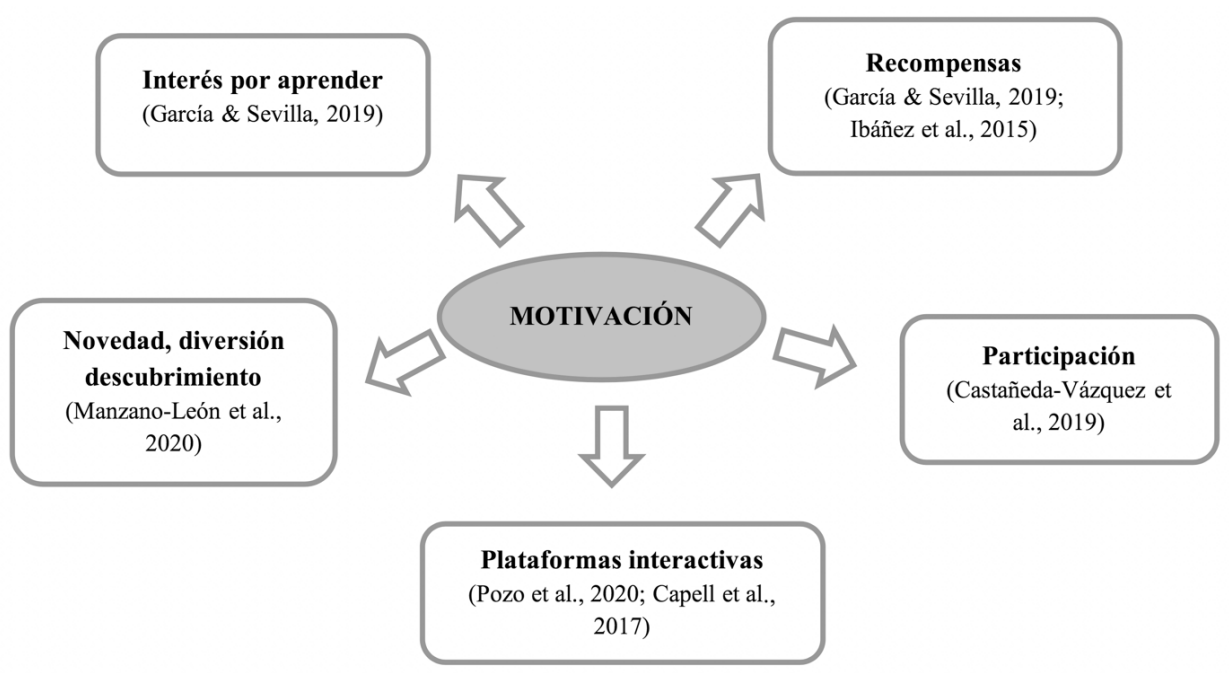

Pozo et al. (2020) relacionan directamente la motivación con la oportunidad de participación activa y el uso de plataformas interactivas y recreativas, puesto que la tecnología se transforma en la lengua vehicular a través de la que se desarrollan algunos sistemas gamificados (Gil y Prieto, 2019). Para Capell et al. (2017) el disfrute 
que proporcionan los videojuegos y las plataformas inciden me manera decisiva en la motivación y el aprendizaje. No obstante, en el extremo opuesto encontramos experiencias en las que se prescinde de dispositivos digitales, logrando un impacto notable en la motivación del alumnado que repercute positivamente en otros aspectos como la autoconfianza, el rendimiento o las relaciones sociales (Quintanal, 2016).

En suma, "la motivación es el motor que mueve al estudiante a reflexionar, investigar o crear sus propias propuestas" (Pérez-López et al., 2017, p. 256), aumentando sus expectativas y descubriendo su potencial (López-Belmonte et al., 2020). De este modo, se configura como un elemento indisociable del proceso de enseñanza y aprendizaje, que actúa como catalizador del interés de los estudiantes y guía su acción hacia la construcción y adquisición de conocimientos.

\section{Compromiso (C)}

El compromiso con el aprendizaje nace de una fuerza interior y responde a un interés natural por seguir avanzando y completar los procesos formativos en los que el sujeto está inmerso. Este elemento no suele contemplarse como un objetivo directo en las propuestas de gamificación, pero sí se reconoce como una de sus ventajas.

La implicación y la persistencia en el trabajo tras haber obtenido la puntuación máxima es uno de los principales indicadores de compromiso. Estos resultados se han constatado en proyectos gamificados, en los que los estudiantes mantuvieron la participación en las actividades después de conseguir los objetivos marcados, superando las expectativas iniciales y el tiempo establecido en el programa educativo (Ibáñez et al., 2015; Díaz-Lauzurica y Moreno-Salinas, 2019).

Esta predisposición a la participación se ve alentada por la motivación (ManzanoLeón et al., 2020; López-Belmonte, 2020), por el componente lúdico y los retos reales (Chou, 2016), que inundan emocionalmente al sujeto hasta alcanzar un elevado nivel de compromiso (Quintanal, 2016) y, en consecuencia, una actitud más positiva hacía las pruebas de evaluación (Díaz-Lauzurica y Moreno-Salinas, 2019).

Por su parte, los sistemas gamificados que son abordados mediante espacios virtuales y entornos inmersivos registran una alta dedicación y permanencia del usuario en el mismo, lo que demuestra su implicación y compromiso (García-Cabot et al., 2020; Faya y Martín-Macho, 2019; Gómez, 2019; Cózar-Gutierrez y Sáez-López, 2016). De cualquier forma, los componentes propios del juego (puntos, niveles, insignias...) suponen un estímulo para dedicar más tiempo a la actividad y fomentar la participación, sean cual sean los recursos empleados (Ortega y Gómez, 2019; FusterGuilló et al., 2019).

Asimismo, la organización interna y el enfoque pedagógico que reciben las prácticas gamificadas son de gran relevancia. Ríos et al. (2019) afirman que la evaluación continua y los diseños tales como los concursos incentivan a los estudiantes para repasar los contenidos y preparar con mayor antelación los exámenes o pruebas. Igualmente, cuando las prácticas son grupales y asumen roles, los estudiantes se ven alentados para mantenerse al día y avanzar en el temario (Álvaro-Tordesillas et al., 2020). Todo ello hace que las tasas elevadas de alumnos que se presentan a las pruebas en la primera convocatoria — coincidiendo con los participantes de proyectos de gamificación-, constituyan un índice alto de compromiso (Castañeda-Vázquez et al., 2019). 
Según Cuevas-Martínez et al. (2019) la asistencia a clase y la entrega de tareas son una muestra fehaciente de compromiso en el alumnado, constatando en su experiencia gamificada un incremento del $90 \%$ en el número de trabajos presentados. Estos resultados son fruto de la motivación y del trabajo continuo, que a su vez influyen en las notas finales.

Gráfico 6. Factores asociados al compromiso

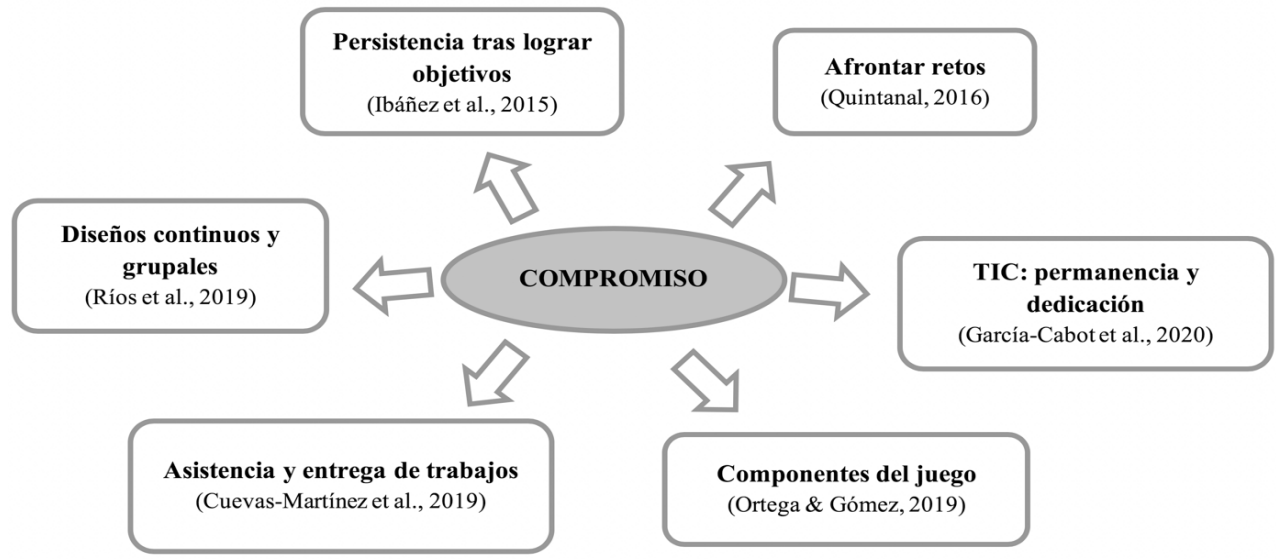

Cohesión grupal (CG)

Las dinámicas y los componentes propios de la gamificación (puntos, tablas de clasificación, competición) pueden percibirse como un peligro para el proceso educativo y las relaciones (Ortega y Gómez, 2019), sin embargo, como apuntan López et al. (2019), el diseño adecuado y la condición lúdica de los sistemas gamificados conducirán hacia la cooperación y el trabajo en equipo.

La formación de grupos heterogéneos con un objetivo común lleva a los estudiantes a colaborar y comunicarse entre sí para obtener insignias, lo que mejora la interacción entre los iguales y las relaciones sociales (López-Belmonte et al., 2020). Las misiones conjuntas, los enigmas y la adquisición de puntos para el avance del equipo se logran mediante las aportaciones de sus miembros (Sipone et al., 2019), de modo que el éxito y el aprendizaje recae en manos de todos los participantes. En este sentido, Vergara et al. (2019) inciden en la importancia de hacer un reparto estratégico de los estudiantes al crear los equipos para garantizar el aprendizaje colaborativo.

Pérez-López et al. (2019) subrayan la relevancia de ofrecer un clima basado en la confianza, pues solo así los implicados se unirán para afrontar los retos y buscar soluciones. La inmersión en este tipo de experiencias genera un sentimiento de pertenencia que hace que los alumnos se sientan pate de una organización, superponiendo el reconocimiento y el éxito del grupo al individual, alcanzando el modelo "Octalysis" definido por Yu-Kai Chou (2016) como la motivación del jugador que se produce por su implicación en el juego al pensar que lo que hace es trascendente. 
La eficacia en el plano social de la gamificación es indudable. Ibáñez et al. (2015) encuentran que algunos estudiantes continúan trabajando con la pretensión de proporcionar a sus compañeros comentarios que les ayuden a continuar aprendiendo y a ganar más puntos. Estas actitudes las califican como altruistas y son un claro ejemplo de cooperación.

Como ya hemos destacado con anterioridad, gran parte de las propuestas de gamificación se complementan con el uso de las TIC. Su utilización refuerza la cohesión grupal, dado que favorecen el trabajo en equipo y la involucración de cada participante en las tareas asignadas para la consecución de los objetivos comunes (Gasca-Hurtado et al., 2015). García-Cabot et al. (2020) abogan por el empleo de plataformas sociales gamificadas, las cuales reportan mejoras en las relaciones y en las habilidades comunicativas.

Pozo et al. (2020) coinciden en el potencial de las TIC dentro de sistemas gamificados para estimular el aprendizaje cooperativo y las interacciones entre los agentes que intervienen. Asimismo, reconoce la oportunidad que brindan a los estudiantes para abordar los problemas desde distintos puntos de vista y nutrirse de otras perspectivas. No obstante, su efectividad depende del planteamiento metodológico, en este caso siguiendo los principios y directrices de la gamificación (Vergara et al., 2019).

En síntesis, se ha comprobado que las actividades gamificadas fomentan la predisposición para trabajar en grupo, produce una mejora en las habilidades sociales (Quintanal, 2016; Capel et al., 2017; Ortega y Gómez, 2019), en la capacidad dialógica (Carrión, 2019) y en otras destrezas que los alumnos desconocían hasta el momento (Álvaro-Tordesillas et al., 2020).

Gráfico 7. Factores asociados a la cohesión grupal

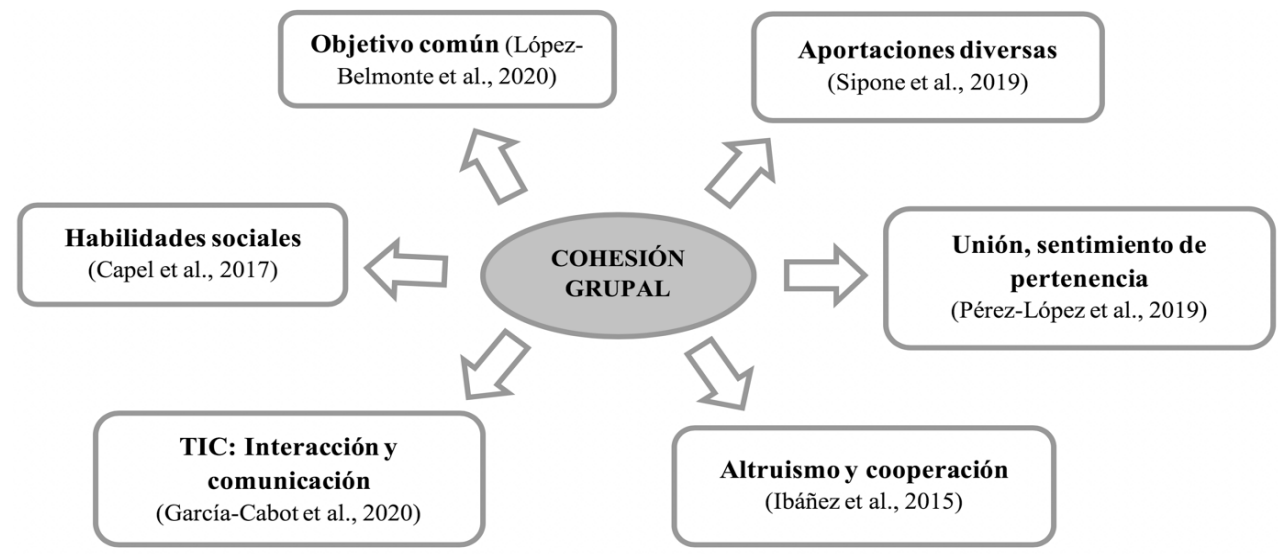

\section{Emociones $(E)$}

Los usuarios de sistemas gamificados pueden experimentar un amplio elenco de sentimientos y emociones a lo largo de todo el proceso, dependiendo de los logros 
y de las dificultades con las que se encuentren para superar los desafíos. Esta condición, a su vez, incidirá en variables como el rendimiento o la cohesión grupal.

Pérez-López et al. (2019) matizan que los retos provocan incertidumbre y que la búsqueda fallida de soluciones puede generar frustración y decepción. Del mismo modo, indican que, derivado de la cohesión grupal los sujetos son capaces de emocionarse por los éxitos del equipo y recomponerse tras los fracasos para seguir intentando hallar la respuesta correcta. Por el contrario, en otros estudios se ha identificado la sensación de desafío para superar obstáculos como una emoción agradable (Ibáñez et al., 2015; Rojo et al., 2019) y como una oportunidad para mejorar la tolerancia del error considerándola parte natural del aprendizaje (Ortega y Gómez, 2019).

Díez et al. (2017) analizan el estado de ánimo de los estudiantes en una propuesta gamificada. Los datos son muy positivos, la mayoría afirman sentirse animados, contentos, tranquilos y optimista; lo que repercute favorablemente en la adquisición y consolidación de contenidos y en la resolución de los ejercicios. En la misma línea, Parra-González et al. (2020) Ilevan a cabo la medición de los niveles de ansiedad ante el aprendizaje, detectando valores más bajos al aplicar técnicas gamificadas; esto se traduce en un mayor entusiasmo y predisposición para aprender. Quintanal (2016) percibe la mejora en la autoestima y la autoconfianza, mientras que QuintasHijós et al. (2020) hablan de un sentimiento positivo general.

Gráfico 8. Factores asociados a las emociones

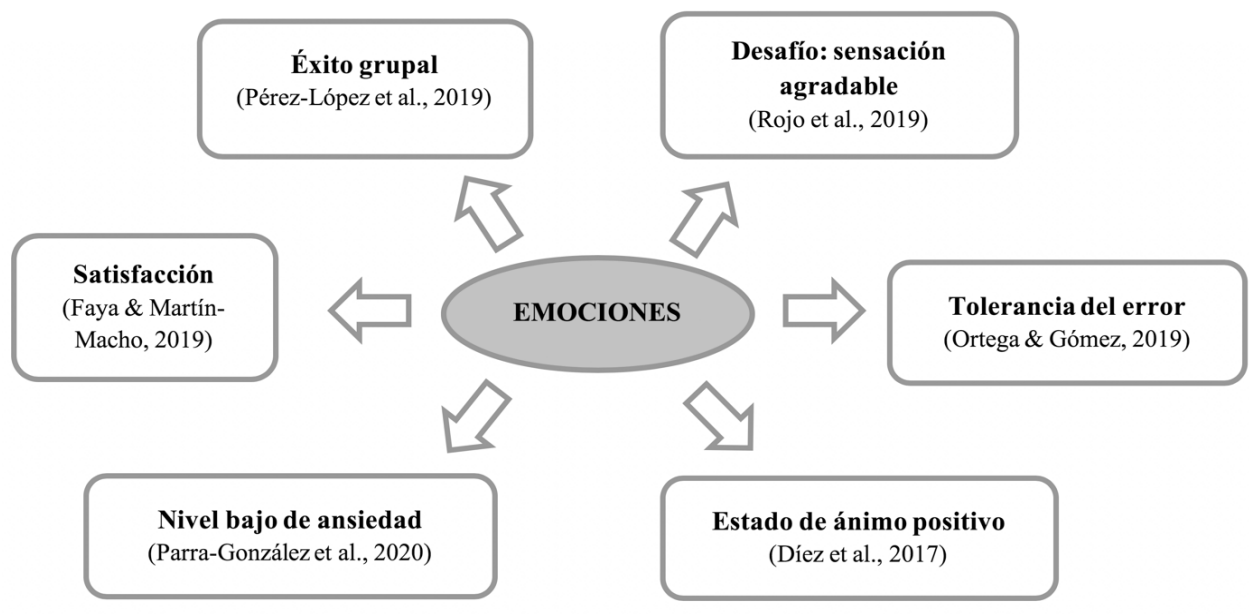

En el marco de la enseñanza universitaria, Zamora-Polo et al. (2019) investigan los parámetros emocionales relacionados con el estudio de las ciencias mediante unidades gamificadas. Los resultados arrojan valores más altos para las emociones positivas, aunque aclara que estas se asocian a la metodología más que a los contenidos de conocimiento. Siguiendo esta dirección, Sánchez-Martín et al. (2018) exploran la influencia del método de enseñanza sobre el desempeño emocional del alumnado. Para ello establecen una diferenciación entre emociones dinámicas y estáticas, positivas y negativas, siendo la gamificación y las actividades prácticas las que se asocian 
en mayor medida con las emociones dinámicas. Otro dato destacable es que los estudiantes identifican el método como la razón principal que determina su estado emocional frente al aprendizaje.

El ambiente distendido y la metodología activa que reside en los enfoques gamificados desencadenan una sensación de control y responsabilidad en los discentes (Pérez-López et al., 2017), reconociendo que se trata de una experiencia muy gratificante que produce una elevada satisfacción general (Faya y Martín-Macho, 2019; Arufe, 2019; Fuester-Guilló et al., 2019; Rojo et al., 2019).

\section{Rendimiento académico $(R A)$}

El éxito del proceso de enseñanza y aprendizaje se identifica, principalmente, con el rendimiento académico de los estudiantes. En cualquier propuesta educativa se contempla como el factor más relevante, a pesar de ello, encontramos que en los sistemas gamificados es la variable que entraña mayor controversia.

García-Cabot et al. (2020) desarrollan un proyecto de gamificación a través de una plataforma social obteniendo mejoras parciales en el rendimiento. Si bien es cierto que perciben un impacto en la adquisición de conocimientos básicos y en determinadas habilidades, no se registran puntuaciones más altas en todas las tareas. De Marcos et al. (2016) añaden que la naturaleza colaborativa de las redes sociales gamificadas rinde beneficios en términos de rendimiento a corto plazo, es decir, producen un efecto más rápido que los enfoques que solo se basan en gamificación. Igualmente, verifican que las experiencias gamificadas no reportan mejoras cuando se trata de una evaluación conceptual, pero sí se observan ventajas cuando se orientan hacia la adquisición de habilidades prácticas.

La introducción de la gamificación para complementar las clases teóricas y prácticas es valorada positivamente por los estudiantes que se ve reflejado en el aumento de las calificaciones (Quintanal, 2016; Capell et al., 2017; Fuster-Guilló et al., 2019), en el rendimiento académico a través de la inteligencia emocional y la motivación (Pozo-Rico y Sandoval, 2020), al desarrollo en la capacidad de aprendizaje, su significatividad y predisposición favorable hacia el aprendizaje (Castañeda-Vázquez et al., 2019; GómezEjerique y López-Cantos, 2019; Parra-González et al., 2020), o unida a recursos trasnsmedia (Gómez-Triguero et al., 2017). Todo ello viene determinado por la influencia del método sobre la comprensión de los contenidos, la profundización y adquisición de conocimientos y el refuerzo del aprendizaje (Ibáñez et al., 2015; Ortega y Gómez, 2019).

Para Díaz-Lauzurica y Moreno-Salinas (2019) la mejora cualitativa en el progreso de los estudiantes y el aumento de las calificaciones son fruto del avance en el razonamiento y la adquisición de aprendizajes significativos que emergen al afrontar los desafíos. Sipone et al. (2019) declaran que la gamificación facilita la comprensión de los contenidos y la identificación y resolución de problemas; convirtiéndose en una metodología imprescindible para introducir temas nuevos y complejos.

Carrión (2019) considera que la gamificación ofrece un aprendizaje de mayor calidad ligado a la adquisición de competencias y a la construcción cooperativa del conocimiento. Esta última idea es compartida por Pozo et al. (2020), quienes valoran la forma dinámica en que se presentan los contenidos como requisito fundamental para la mejora de las calificaciones. 
Los hallazgos de Sánchez-Rivas y Ruiz-Palmero (2019) al comparar las pruebas de evaluación tradicionales y gamificadas no siguen la norma hasta ahora expuesta, ya que las diferencias en término de rendimiento no son sustanciales. Sin embargo, reconocen su contribución al desarrollo de competencias clave y un aprendizaje más enriquecedor durante la prueba. Asimismo, López et al. (2019) ultiman en su investigación que la gamificación no asegura un mejor aprendizaje, pero sí un desarrollo competencial superior al de una práctica convencional.

Los cambios pedagógicos que abordan estas propuestas, como las evaluaciones formativas, el feedback continuado o la adaptación del proceso de enseñanza y aprendizaje a los rasgos culturales de los millennials, se traducen en calificaciones más altas (Ríos et al., 2019). Otra de las grandes ventajas de la gamificación es el compromiso de los estudiantes con las tareas, variable indisociable de las mejora en el rendimiento; haciendo de estas metodologías un recurso esencial para luchar contra el abandono (Cuevas-Martínez, 2019; Álvaro-Tordesillas et al., 2020).

Gráfico 9. Factores asociados al rendimiento académico

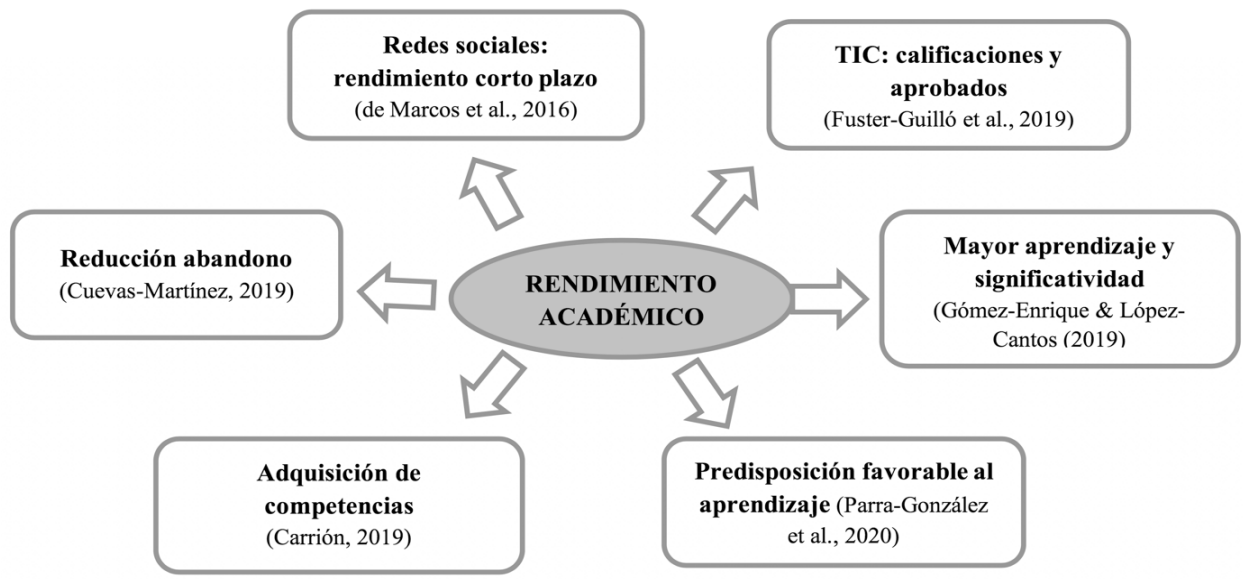

\section{Categoría central: La gamificación como estímulo para el aprendizaje y el desarrollo social y personal}

El análisis cualitativo de los artículos seleccionados nos ha permitido determinar los efectos positivos de la gamificación en el proceso de enseñanza y aprendizaje. A partir de los mismos, hemos enunciado 5 categorías (motivación, compromiso, cohesión grupal, emociones y rendimiento académico) entre las que se establecen conexiones directas.

A continuación presentamos un gráfico que resume la red descriptiva causal existente entre las variables de esta investigación:

Como se puede observar en el esquema, el rendimiento académico se encuadra en el eje central, congregando a su alrededor el resto de las variables. Esto deriva del efecto decisivo que tienen la motivación, el compromiso, la cohesión grupal y las emociones sobre el aprendizaje y los resultados de los estudiantes. 
Gráfico 10. Red descripitiva causal entre las variables

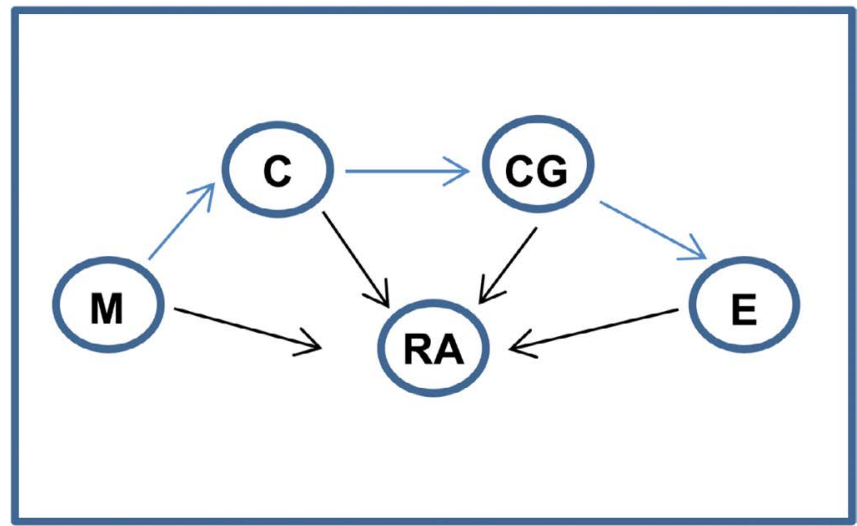

En segundo lugar, percibimos una relación lineal entre motivación, compromiso, cohesión grupal y emociones, basada en un proceso de retroalimentación continuo que desencadena actitudes y acciones deseables en el alumnado.

Las atribuciones que justifican la presencia de cada categoría y su relevancia quedan redactadas en el discurso, reflejando su peso en los sistemas gamificados y dando lugar a la creación de una nueva categoría central que reúne a todas las anteriores. Esta se ha denominado "la gamificación como estímulo para el aprendizaje y el desarrollo social y personal", ya que consideramos que el conglomerado de las 5 variables iniciales puede representarse mediante un código genérico que resuma las potencialidades de la gamificación.

Gráfico 11. Relaciones entre las categorías

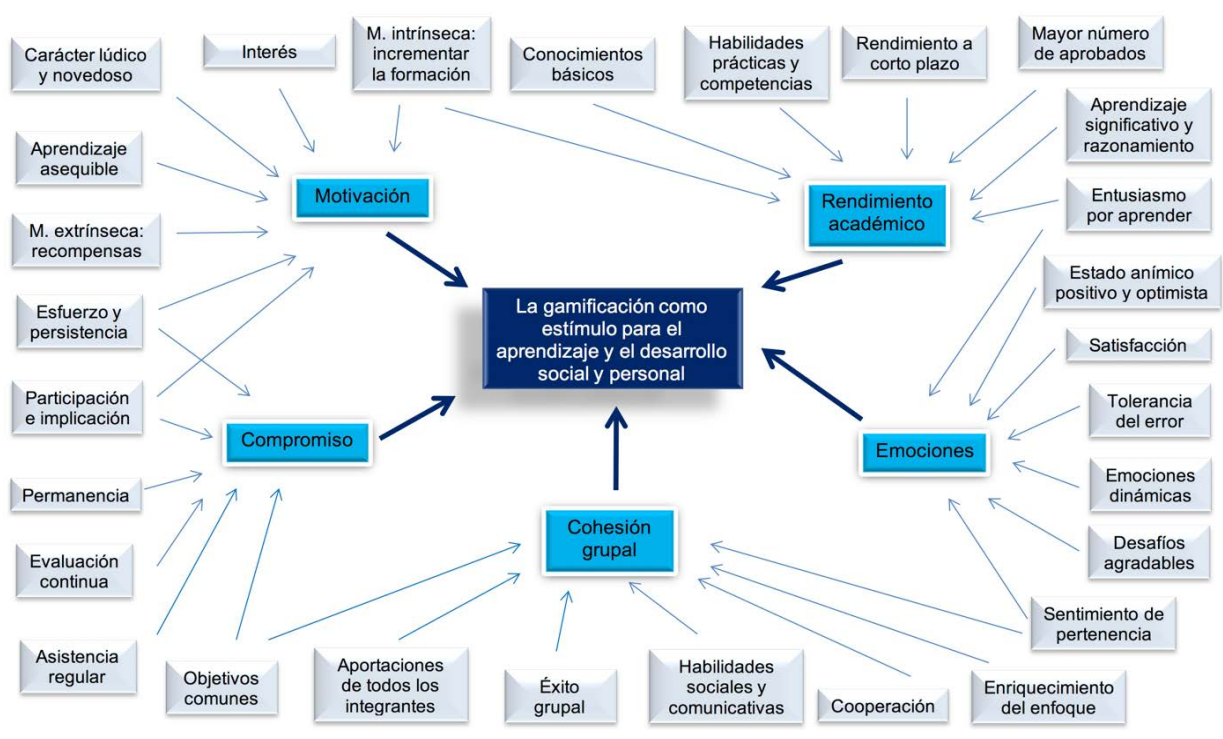




\section{Conclusiones, discusión y futuras líneas de investigación}

Los resultados obtenidos tras la revisión sistemática de los documentos seleccionados confirman el objetivo general de esta la investigación. La aplicación de la gamificación en la educación formal tiene un impacto positivo en el proceso de enseñanza y aprendizaje. Esta afirmación se justifica por los datos generalmente consensuados en los artículos analizados sobre experiencias gamificadas en el aula, en los que no percibimos contraindicaciones ni diferencias muy significativas en los resultados.

De esta declaración se deducen las siguientes conclusiones:

Aplicación de nuevas metodologías que conecten con la forma que tiene de aprender y procesar la información el estudiante del siglo XXI.

- El alumno de hoy adopta un papel activo en su vida y en la educación, desarrollando destrezas ligadas al descubrimiento, la exploración, la creación y la transformación de productos; es decir, no necesitan recibir y memorizar cantidades ingentes de información, sino ser correctamente estimulados para descubrir y actuar por sí mismos. En este contexto, la gamificación reúne los ingredientes básicos para convertir a los discentes en protagonistas y constructores de sus conocimientos (Pérez-López et al., 2017).

- Las estrategias de gamificación surten un efecto positivo en el aprendizaje y en el desarrollo integral del individuo. Estos beneficios pueden identificarse con el conjunto de parámetros extraídos en el presente trabajo, entre los que prevalece una estrecha relación con similares efectos positivos en los comportamientos y resultados en otros contextos de índole no educativo (Zichermann y Linder, 2013).

- La motivación es la variable más recurrente en las experiencias de gamificación, su presencia e incremento es inequívoco. Las dos dimensiones que encierra - extrínseca e intrínseca- se ven favorecidas y perfectamente asociadas con los elementos propios de la gamificación como así los atestiguan Quintero et al. (2018) y Oda Domínguez (2020). Por un lado, los puntos, insignias y recompensas ejercen una influencia externa sobre el individuo; por otro lado, la novedad, el placer o los desafíos canalizan el interés interno. Su conjunción despierta la curiosidad y capta la atención del sujeto, desencadenando la implicación en la propuesta educativa y, por ende, afectando a su rendimiento académico.

- El compromiso se configura sobre la base de la motivación y se traduce en el esfuerzo y la persistencia. Cuando los estudiantes participan en un proyecto gamificado manifiestan niveles altos de interés y una actitud positiva hacia el aprendizaje, siendo constantes en sus tareas. Además, la formación de equipo tan habitual en este enfoque refuerza la responsabilidad para con los otros, alentando a un trabajo continuado, efecto que también producen los puntos y reconocimientos. Todo ello, estimula al estudiante para prepararse los contenidos, realizar las actividades, repasar, indagar... aumentando las posibilidades de éxito en los resultados.

- La cohesión grupal, entendida como el vínculo entre los integrantes de un equipo, es otra de las potencialidades de este enfoque metodológico. Su esencia radica en el esfuerzo compartido para alcanzar un objetivo común. El hecho de 
afrontar desafíos, resolver problemas y avanzar en las tablas de clasificación de manera conjunta constituye un punto de encuentro entre los alumnos del mismo grupo. La colaboración con los compañeros y el intercambio comunicativo procuran mejoras en el rendimiento, en las habilidades sociales y generan un sentimiento de pertenencia que hace más agradable el aprendizaje.

- El proceso de enseñanza y aprendizaje suscita emociones y sentimientos en los sujetos. En el caso de la gamificación, la resolución de problemas y desafíos se percibe como un reto positivo y una posibilidad de superación que infunde ánimos a los participantes para esforzarse por sortear los obstáculos y llegar a la meta. Asimismo, las prácticas grupales desencadenan emociones compartidas, de manera que las derrotas son mejor asimiladas y los éxitos más gratos. El optimismo y el entusiasmo, que subyacen a niveles bajos de ansiedad, hacen que los estudiantes se involucren activamente en las tareas mejorando la adquisición de conocimientos y, en consecuencia, experimentando una grata sensación de satisfacción.

- El rendimiento académico es una variable que aglutina disparidad en los resultados, lo cual puede atribuirse a la amplitud de las muestras, el tipo de experiencias y a las escasas investigaciones con grupo control y experimental. Esto dificulta la generalización de los hallazgos y el consenso respecto a su potencial. Específicamente, podemos hablar de una dicotomía en los datos: en primer orden, un compendio de estudios reconoce mejoras en términos cuantitativos, esto es, un aumento en las calificaciones y en las tasas de aprobados; en segunda instancia, otros trabajos apuntan hacia un mayor desarrollo de habilidades prácticas y competencias clave, sin que se deriven, necesariamente, puntajes más altos. En suma, la ilación depende de la lectura que hagamos de la información, si valoramos el progreso cualitativo y cuantitativo podemos afirmar que la gamificación es efectiva para incrementar el rendimiento académico.

- El conjunto de ventajas que reconocemos en la gamificación educativa conduce a la configuración de una categoría central, a la que denominamos "la gamificación como estímulo para el aprendizaje y el desarrollo social y personal". Como acabamos de explicitar, esta estrategia favorece el desarrollo de distintos ámbitos del individuo. Promueve el descubrimiento y adquisición de contenidos; el desarrollo de destrezas y competencias; la interacción social y el intercambio comunicativo; el reconocimiento de emociones, su expresión y dominio; el esfuerzo personal y el gusto por aprender.

- Finalmente, determinar que la gamificación constituye una estrategia innovadora aplicable a las distintas etapas de la educación formal que reúne un potencial considerable para contribuir a la reducción de tasas de abandono escolar y a la transformación del sistema, y que requiere de un gran diseño instructivo que se adapte a las características del juego (Kapp, 2012).

De cara a futuras investigaciones sería interesante valorar la influencia de las tecnologías avanzadas en los sistemas gamificados. En este trabajo no se ha contemplado como objetivo, pero hemos observado su alta incidencia en las diferentes propuestas y algunas de las oportunidades educativas que encierra. 


\section{REFERENCIAS BIBLIOGRÁFICAS}

Alomari, I., Al-Samarraie, H. y Yousef, R. (2019). The role of gamification techniques in promoting student learning: A review and synthesis. Journal of Information Technology Education: Research, 18, 395-417. https://doi.org/10.28945/4417

[*] Álvaro-Tordesillas, A., Alonso-Rodríguez, M., Poza-Casado, I. y Galvan-Desvaux, N. (2020). Gamification experience in the subject of Descriptive Geometry for Architecture. Educación XX1, 23(1), 373-408. https://doi.org/10.5944/ educxx1.23591

[*] Arufe, V. (2019). Fortnite EF un nuevo juego deportivo para el aula de Educación Física. Propuesta de innovación y gamificación basada en el videojuego Fortnite. Sportis Scientific Technical Journal, 5(2), 323-350. https://doi.org/10.17979/ sportis.2019.5.2.5257

Burke, B. (2014). Gamify: How Gamification Motivates People to do Extraordinary Things. Bibliomotion.

[*] Capell, N., Tejada, J. y Bosco, A. (2017). Los videojuegos como medio de aprendizaje: Un estudio de caso en matemáticas en educación primaria. PíxelBit. Revista de Medios y Educación, 51, 133-150. http://doi.org/10.12795/ pixelbit.2017.i51.09

Carreras, C. (2017). Del homo ludens a la gamificación. Quaderns de filosofía 4(1), 107-118. http://doi.org/10.7203/qfia.4.1.9461

[*] Carrión, E. (2019). El uso del juego y la metodología cooperativa en la Educación Superior: una alternativa para la enseñanza creativa. Educación y Pedagogía, 23, 70-97. http://dx.doi.org/10.6035/Artseduca.2019.23.4

[*] Castañeda-Vázquez, C., Espejo-Garcés, T., Zurita-Ortega, F. y Fernández-Revelles, A. B. (2019). La formación de los futuros docentes a través de la gamificación, tic y evaluación continua. SPORT TK: Revista Euroamericana de Ciencias del Deporte, 8(2), 55-64. https://doi.org/10.6018/sportk.391751

Chou, Y. K. (2016). Actionable Gamification: beyond points, badges and leaderboards. Octalysis Media.

Colom, A. J. (2005). Continuidad y complementariedad entre la educación formal y no formal. Revista de Educación, 338, 9-22. http://hdl.handle.net/11162/68740

[*] Cózar-Gutiérrez, R. y Sáez-López, J. M. (2016). Game-based learning and gamification in initial teacher training in the social sciences: an experiment with MinecraftEdu. International Journal of Educational Technology in Higher Education, 13, 2. https://doi.org/10.1186/s41239-016-0003-4

[*] Cuevas-Martínez, J. C., Yuste-Delgado, A. J., Pérez-Lorenzo, J. M. y Triviño-Cabrera, A. (2019). Jump to the Next Level: A Four-Year Gamification Experiment in Information Technology Engineering. IEEE Access, 7, 118125-118134 https://doi. org/10.1109/ACCESS.2019.2932803

[*] De-Marcos, L., García-López, E. y García-Cabot, A. (2016). On the effectiveness of game-like and social approaches in learning: Comparing educational gaming, gamification \& social networking. Computers \& Education, 95, 99-113. http://doi. org/10.1016/j.compedu.2015.12.008 
[*] Díaz-Lauzurica, B. y Moreno-Salinas, D. (2019). Computational Thinking and Robotics: A Teaching Experience in Compulsory Secondary Education with Students with High Degree of Apathy and Demotivation. Sustainability 11(18). https://doi. org/10.3390/su11185109

[*] Díez, J. C., Bañeres, D. y Serra, M. (2017). Experiencia de gamificación en Secundaria en el Aprendizaje de Sistemas Digitales. Education in the Knowledge Society, EKS, 18(2), 85-105. https://doi.org/10.14201/eks201718285105

Dorio, I., Sabariego, M. y Massot, I. (2004). Características generales de la metodología cualitativa. En R. Bisquerra (Ed.), Metodología de la Investigación Educativa (pp. 275-292). La Muralla.

Escaravajal, J. C. y Martín-Acosta, F. (2019). Análisis bibliográfico de la gamificación en Educación Física. Revista Iberoamericana de Ciencias de la Actividad Física y el Deporte, 8(1), 97-109. https://doi.org/10.24310/riccafd.2019.v8i1.5770

[*] Faya, F. y Martín-Macho, A. (2019). Socrative in Higher Education: Game vs. Other Uses. Multimodal Technologies and Interaction, 3(3), 49. https://doi.org/10.3390/ mti3030049

[*] Fuster-Guilló, A., Pertegal-Felices, M. L., Jimeno-Morenilla, A. Azorín-López, J., Rico-Soliveres, M. L. y Restrepo-Calle, F. (2019). Evaluating Impact on Motivation and Academic Performance of a Game-Based Learning Experience Using Kahoot. Frontiers Psychology, 10, 2843. https://doi.org/10.3389/fpsyg.2019.02843

[*] García-Cabot, A., García-López, E., Caro-Álvaro, S., Gutiérrez-Martínez, J. M. y de-Marcos, L. (2020). Measuring the effects on learning performance and engagement with a gamified social platform in an MSc program. Comput Appl Eng Educ, 28, 207-223. https://doi.org/10.1002/cae.22186

[*] García, A. y Sevilla, S. (2019). El método IBI en la enseñanza de ELE. Aplicación de la gamificación en el Camino de Santiago. Foro de Profesores de E/LE, 15, 243 265. http://doi.org/10.7203/foroele.15.16027

Gallardo, J. A. y Gallardo, P. (2018). Teorías sobre el juego y su importancia como recurso educativo para el desarrollo integral infantil. Revista Educativa Hekademos, 24, 41-51. https://cutt.ly/nyLj6qa

García, A. y Llull, J. (2009). El juego infantil y su metodología. Editex.

García, A. y Sevilla, S. (2019). El método IBI en la enseñanza de ELE. Aplicación de la gamificación en el Camino de Santiago. Foro de Profesores de E/LE, 15, 243-265. http://doi.org10.7203/foroele.15.16027

[*] Gasca-Hurtado, G. C., Peña, A., Gómez-Álvarez, M. C., Plascencia-Osuna, O. A. y Calvo-Manzano, J. A. (2015). Realidad virtual como buena práctica para trabajo en equipo con estudiantes de ingeniería. RISTI - Revista Ibérica De Sistemas $E$ Tecnologías De Información, 16, 76-91. https://cutt.ly/YyKpimj

[*] Gil, J. y Prieto, E. (2019). Juego y gamificación: Innovación educativa en una sociedad en continuo cambio. Revista Ensayos Pedagógicos, 14(1), 91-121. http:// doi.org/10.15359/rep.14-1.5

Gisbert, J. P. y Bonfill, X. (2004). Cómo realizar, evaluar y utilizar revisiones sistemáticas y meta análisis. Gastrenterol Hepatol, 27(3), 129-149. https://cutt.ly/RyLj3rk 
Gómez, J. F. (2012). El juego infantil y su importancia en el desarrollo. CCAP, 10(4), 5-13. https://cutt.ly/dyLj2Fo

Gómez, I. M. (2019). Methodologies Gamified as Didactic Resources for Social Sciences. iJET, 14(23), 193-207. https://doi.org/10.3991/ijet.v14i23.10794

[*] Gómez-Carrasco, C. J., Monteagudo-Fernández, J., Moreno-Vera, J. R. y SainzGómez, M. (2019). Effects of a Gamification and Flipped-Classroom Program for Teachers in Training on Motivation and Learning Perception. Education Science, 9(4). https://doi.org/10.3390/educsci9040299

[*] Gómez-Ejerique, C. y López-Cantos, F. (2019). Application of innovative teachinglearning methodologies in the classroom. Coaching, flipped-classroom and gamification. A case study of success. MUSE, 6(1), 46-70. https://doi.org/10.4995/ muse.2019.9959

[*] Gómez-Trigueros, I. M., Rovira-Collado, J. y Ruiz-Bañuls, M. (2018). Literatura e Historia a través de un universo transmedia: posibilidades didácticas del Ministerio del Tiempo. Revista Mediterránea de Comunicación/Mediterranean Journal of Communication, 9(1), 217-225. https://doi.org/10.14198/MEDCOM2018.9.1.18

Granda-Orive, J., Alonso-Arroyo, A., García-Río, F., Solano-Reina, S., JiménezRuiz, C. y Aleixandre-Benavent, R. (2013). Ciertas ventajas de Scopus sobre Web of Science en un análisis bibliométrico sobre tabaquismo. Revista española de Documentación Científica, 36(2). https://doi.org/10.3989/redc.2013.2.941

Hernández, R., Fernández, C. y Baptista, P. (2010). Metodología de la investigación. Mcgraw-Hill.

Herranz, E. y Colomo-Palacios, R. (2012). La Gamificación como agente de cambio en la Ingeniería del Software. Revista de Procesos y Métricas, 9(2), 30-56. https:// cutt.ly/CyLkRYv

Ibáñez, E., Cuesta, M., Taglibaue, R. y Zangaro, M. (2008). La generación actual en la universidad: el impacto de los millennials. V Jornadas de Sociología de la UNLP. Universidad Nacional de La Plata. Facultad de Humanidades y Ciencias de la Educación. Departamento de Sociología. https://cutt.ly/fyLIImP

Ibáñez, M. B., Delgado-Kloos, C. y Di-Serio, A. (2014). Gamification for Engaging Computer Science Students in Learning Activities: A Case Study. IEEE Transactions on Learning Technologies, 7(3), 291-301. https://cutt.ly/zyLhL6R

Kapp, K. (2012). The Gamification of Learning and Instruction: Game-Based Methods and Strategies for Training and Education. Pfeiffer.

Latorre, A., Rincón, D. y Arnal, J. (2003). Bases metodológicas de la investigación educativa. Ediciones Experiencia.

[*] Llorens, F., Gallego, F. J., Villagrá, C. J., Compañ, P., Satorre, R. y Molina, R. (2016). Gamification of the learning process: lessons learned. IEEE Revista Iberoamericana de tecnologías del aprendizaje, 11(4), 227-234. http://doi.org/10.1109/ RITA.2016.2619138

López, D., Calonge, A., Rodríguez, T., Ros, G. y Lebrón, J. A. (2019). Using Gamification in a Teaching Innovation Project at the University of Alcalá: A New Approach to Experimental Science Practices. The Electronic Journal of e-Learning, 17(2), 93106. https://cutt.ly/4yLhBLT 
[*] López-Belmonte, J., Segura-Robles, A., Fuentes-Cabrera, A. y Parra-González, M. E. (2020). Evaluating Activation and Absence of Negative Effect: Gamification and Escape Rooms for Learning. International Journal Environmental Research and Public Health, 17(7). https://doi.org/10.3390/ijerph17072224

Lozada-Ávila, C. y Betancur-Gómez, S. (2017). La gamificación en la educación superior: una revisión sistemática. Revista Ingenierías Universidad de Medellín, 16, 31. http://doi.org/10.22395/rium.v16n31a5

[*] Manzano-León, A., Sánchez-Sánchez, M., Trigueros-Ramos, R., Álvarez-Hernández, J. y Aguilar-Parra, J. M. (2020). Gamificación y Breakout Edu en Formación Profesional. El programa "Grey Place" en Integración Social. EDMETIC, Revista de Educación Mediática y TIC, 9(1), 1-20. https://doi.org/10.21071/edmetic.v9i1.12067

Marczewski, A. (2015). Even Ninja Monkeys Like to Play: Gamification, Game Thinking and Motivational Design. CreateSpace Independent Publishing Platform.

Oda Domínguez, H. (2020). La gamificación: una revisión sistemática y proyecto innovador con relación a la motivación y percepción subjetiva del esfuerzo. Trabajo de Fin de Máster. Universidad de La Laguna. https://riull.ull.es/xmlui/ handle/915/19788

Oliva, H. A. (2016). La gamificación como estrategia metodológica en el contexto educativo universitario. Realidad y reflexión, 44, 29-47. https://cutt.ly/AyLk0Uk

[*] Ortega, D. y Gómez, I. M. (2019). Gamification, social problems, and gender in the teaching of social sciences: Representations and discourse of trainee teachers. PLOS ONE, 14(6), e0218869. https://doi.org/10.1371/journal.pone.0218869

[*] Ortiz-Colón, A. M., Jordán, J. y Agredal, M. (2018). Gamificación en educación: una panorámica sobre el estado de la cuestión. Educ. Pesquisa, 44(23), 1-17. https://doi.org/10.1590/s1678-4634201844173773

Paniagua, G. y Palacios, J. (2008). Respuesta a la diversidad educativa. Alianza.

[*] Parra-González, M. E., López, J., Segura-Robles, A. y Fuentes, A. (2020). Active and Emerging Methodologies for Ubiquitous Education: Potentials of Flipped Learning and Gamification. Sustainability, 12(2). https://doi.org/10.3390/su12020602

[*] Pascuas, Y. S., Vargas, E. O. y Muñoz, J. I. (2017). Experiencias motivacionales gamificadas: una revisión sistemática de literatura. Innovación educativa, 17(75), 63-80. https://cutt.ly/MyLlj7g

[*] Pérez-López, I. J., Rivera, E. y Trigueros, C. (2019). 12 +1. Sentimientos del alumnado universitario de educación física frente a una propuesta de gamificación: "Game of thrones: la ira de los dragones". Movimiento, 25. https://doi. org/10.22456/1982-8918.88031

Pérez-López, I.J., Rivera, E. y Trigueros, C. (2017). La profecía de los elegidos": un ejemplo de gamificación aplicado a la docencia universitaria. Revista Internacional de Medicina y Ciencias de la Actividad Física y el Deporte, 17(66), 243-260. https://doi.org/10.15366/rimcafd2017.66.003

[*] Pozo, S., Belmonte, J. M., Fuentes, A. y López J. A. (2020). Gamification as a Methodological Complement to Flipped Learning-An Incident Factor in Learning Improvement. Multimodal Technologies and Interaction, 4(2), 12. https://doi. org/10.3390/mti4020012 
[*] Pozo-Rico, T. y Sandoval, I. (2020). Can Academic Achievement in Primary School Students Be Improved Through Teacher Training on Emotional Intelligence as a Key Academic Competency? Frontiers Psychol, 10, 2976. https://doi.org/10.3389/ fpsyg.2019.02976

Prensky, M. (2001). Digital natives, digital inmigrants. On the horizon, NBC University Press, 9(5). https://cutt.ly/jlrnSuZ

PRISMA-Statement website (2015). http://www.prisma-statement.org

Piantanida, M. y Garman, N. B. (1999). The Qualitative Dissertation: A Guide for Students and Faculty. Corwin Press, INC.

Prieto, J. M. (2020). Una revisión sistemática sobre gamificación, motivación y aprendizaje en universitarios. Teoría de la Educación. Revista interuniversitaria, 32(1), 73-99. http://doi.org/10.14201/teri.20625

[*] Quintanal, F. (2016). Gamificación y Física y Química en Educación Secundaria. Education in the Knowledge Society, EKS, 17(3), 13-28. https://doi.org/10.14201/ eks20161731328

Quintas-Hijós, A., Peñarrubia-Lozano, C. y Bustamante J. C. (2020). Analysis of the applicability and utility of a gamified didactics with exergames at primary schools: Qualitative findings from a natural experiment. PLOS ONE, 15(4). https://doi. org/10.1371/journal.pone.0231269

Quintero, L., Jiménez, F. y Area, M. (2018). Más allá del libro de texto. La gamificación mediada con TIC como alternativa de innovación en educación física. Retos, 34, 343-348.

[*] Ríos, A., Muñoz, I., Castro, P. y Arroyo, J. I. (2019) Gamificación, estrategia compartida entre universidad, empresa y millennials. [Gamification, strategy shared between university, company and millennials]. REDU. Revista de Docencia Universitaria, 17(2), 73-88. https://doi.org/10.4995/redu.2019.11479

Rodrigues, R. J., Gouveia, R. y Pereira, C. T. (2019). Gamification in Management Education: A Systematic Literature Review. BAR - Brazilian Administration Review 16(2). http://doi.org/10.1590/1807-7692bar2019180103

Rodríguez, D. y Valldeoriola, J. (2009). Metodología de la investigación. Universitat Oberta de Cataluña. https://cutt.ly/qyLle5Z

[*] Rojo, T., González-Limón, M. y Rodríguez-Ramos, A. (2019). Company-University Collaboration in Applying Gamification to Learning about Insurance. Informatics 6(3). https://doi.org/10.3390/informatics6030042

[*] Sánchez-Martin, J., Cañada-Cañada, F. y Dávila-Acedo, M. A. (2018). Emotional responses to innovative science teaching methods: acquiring emotional data in a general science teacher education class. JOTSE, 8(4), 346-359. https://doi. org/10.3926/jotse.408

[*] Sánchez-Rivas, E., Ruiz-Palmero, J. y Sánchez-Rodríguez, J. (2019). Gamification of Assessments in the Natural Sciences Subject of Primary Education. Educational Sciences: Theory \& Practice, 19(1), 95-111. http://doi.org/10.12738/ estp.2019.1.0296

Savin-Baden, M. y Major, C. (2013) Qualitative research: The essential guide to theory and practice. Routledge, London. 
[*] Sipone, S., Abella-García, V., Barreda, R. y Rojo, M. (2019). Learning about Sustainable Mobility in Primary Schools from a Playful Perspective: A Focus Group Approach. Sustainability, 11(8), 2387. https://doi.org/10.3390/su11082387

[*] Vergara, D., Mezquita, J. M. y Gómez, A. I. (2019). Metodología innovadora basada en la gamificación educativa: Evaluación tipo test con la herramienta Quizizz. Profesorado. Revista de Currículum y Formación de Profesorado, 23(3), 363-387. https://doi.org/10.30827/profesorado.v23i3.11232

[*] Villalustre, L. y del Moral, M. E. (2015). Gamificación: estrategias para optimizar el proceso de aprendizaje y la adquisición de competencias en contextos universitarios. Digital Education Review, 27, 13-31. https://doi.org/10.1344/der.2015.27.1331

[*] Zamora-Polo, F., Corrales-Serrano, M., Sánchez-Martín, J. y Espejo-Antúnez, L. (2019). Nonscientific University Students Training in General Science Using an Active-Learning Merged Pedagogy: Gamification in a Flipped Classroom. Education Science, 9(4), 297. https://doi.org/10.3390/educsci9040297

Werbach, K. y Hunter, D. (2012). For the win. How game thinking can revolutionize your business. Wharton Digital Press.

Zichermann, G. y Cunningham, C. (2011). Gamification by design. Implementing Game Mechanics in Web and Mobile Apps. O'Reilly Publishing Bibliomotion.

Zichermann, G. y Linder, J. (2013). The Gamification Revolution: How Leaders Leverage Game Mechanics to Crush The Competition. McGraw-Hill. 